\title{
CONCEPT OF gH DIFFERENTIABILITY IN SOLVING SECOND ORDER LINEAR HOMOGENEOUS ODES BASED ON THE RELATION BETWEEN FLT AND ITS $\mathbf{k}^{\text {th }}$ DERIVATIVE FOR THE CASE $k \geq 1$ IN FUZZY ENVIRONMENT
}

\author{
Habib H. ${ }^{*}$, Tahir A. ${ }^{2}$, Musa S. ${ }^{2}$ and Yusuf K. P. ${ }^{3}$ \\ ${ }^{1}$ Department of Mathematics, College of Education Waka-Biu, Borno State, Nigeria \\ ${ }^{2}$ Department of Mathematics, Modibbo Adama University, Yola, Adamawa State, Nigeria \\ ${ }^{3}$ Department of Mathematics, Federal College of Education Technical, Potiskum, Yobe State \\ Nigeria
}

*Corresponding Author's Email: habibkantoma@gmail.com; Phone No.: +2348034463968

\section{Cite this article:}

Habib H., Tahir A., Musa S., Yusuf K.P. (2022), Concept of gH Differentiability in Solving Second Order Linear Homogeneous ODEs Based on the Relation between FLT and Its $\mathrm{k}^{\text {th }}$ Derivative for the Case $k \geq 1$ in Fuzzy Environment. African Journal of Mathematics and Statistics Studies 5(1), 1-13. DOI: 10.52589/AJMSSPZZNOBYY

\section{Manuscript History}

Received: 12 Dec 2021

Accepted: 29 Dec 2021

Published: 12 Jan 2022

Copyright $\odot 2020$ The Author(s). This is an Open Access article distributed under the terms of Creative Commons AttributionNonCommercial-NoDerivatives 4.0 International (CC BY-NC-ND 4.0 ), which permits anyone to share, use, reproduce and redistribute in any medium, provided the original author and source are credited.
ABSTRACT: In this study, a fuzzy Laplace transform is used to solve second order linear homogeneous ordinary differential equations. The solution obtained is based on the concept of $g H$ differentiability and the relation between the fuzzy Laplace transform and its $k^{\text {th }}$ derivative for $k \geq 1$ is obtained. Examples are constructed for the existence and uniqueness of solutions of second order FODE.

KEYWORDS: gH Differentiability, Linear Homogeneous ODE, Fuzzy Laplace Transform. 


\section{INTRODUCTION}

Generalization of the Hukuhara derivative (gH-derivative) based on fuzzy derivative was first defined by Seikkala (1987) who discovered that the fuzzy initial value problem (FIVP)

$$
x^{\prime}(t)=f(t, x(t)), x(0)=x_{0}
$$

has a unique fuzzy solution when $f$ satisfies the generalized Lipschitz condition. Fuzzy differential equations (FDEs) and initial value problems were extensively treated and used in many models by many researchers that included population models by Guo, Xue and Li (2003), Civil Engineering by Oberguggenberger and Pittschmann (1999), Bioinformatics and Computational Biology by Casasnovas and Rossell (2005), and Quantum Optics and Gravity by Naschie (2005). Eljaoui, Melliani and Chadli (2015) solved second order linear homogeneous ODEs based on the concept of generalized differentiability and using Laplace transform in a fuzzy environment. They considered the equation

$$
y^{\prime \prime}(t)=f\left(t, y(t), y^{\prime}\right), y(0)=y_{0}=\left(\underline{y}_{0}, \bar{y}_{0}\right), y^{\prime}(0)=z_{0}=\left(\underline{z}_{0}, \bar{z}_{0}\right)
$$

and assumed it to be linear with respect to $\left(y(t), y^{\prime}(t)\right)$. Based on what is observed in this study, Eljaoui, Melliani and Chadli (2015) work was limited to a certain assumption that the fuzzy linear function

$$
f\left(t, y(t), y^{\prime}(t)\right)=a y(t)+b y^{\prime}(t)+c(t)
$$

is a crisp mapping but the relationship between the Fuzzy Laplace transform (FLT) of a fuzzy function and that of its $k^{\text {th }}$ derivative, with $k \geq 1$ were not considered in the work. It is also observed here that in their work, existence and uniqueness of solution were not presented. This study therefore addresses problems associated with work of Eljaoui, Melliani and Chadli (2015) and as well as applies the fuzzy Laplace transformation method to solve a large class of FDEs.

\section{METHOD}

Consider the following second order ODE in fuzzy environment:

$$
\left\{\begin{array}{l}
y^{\prime \prime}(t)=f\left(t, y(t), y^{\prime}\right), \\
y(0)=y_{0}=\left(\underline{y}_{0}, \bar{y}_{0}\right) \in E \\
y^{\prime}(0)=z_{0}=\left(\underline{z}_{0}, \bar{z}_{0}\right) \in E
\end{array}\right.
$$

where

$$
y(t)=(\underline{y}(t, \alpha), \bar{y}(t, \alpha))
$$


is a fuzzy valued function of $f \geq 0$ and $f\left(t, y(t), y^{\prime}(t)\right)$ is a fuzzy valued function, which is linear with respect to $y(t), y^{\prime}(t)$. Applying Laplace transform to equation (1), we have

$$
L\left[y^{\prime \prime}(t)\right]=L\left[f\left(t, y(t), y^{\prime}(t)\right)\right] .
$$

The process of solving equation (1) exists if ${ }^{y}$ and $y^{\prime}$ are (i) ${ }^{\mathrm{gH}}$-differentiable

$$
\begin{aligned}
& y^{\prime}(t)=\left(\underline{y}^{\prime}(t, \alpha), \bar{y}^{\prime}(t, \alpha)\right) \text { and } y^{\prime \prime}(t)=\left(\underline{y}^{\prime \prime}(t, \alpha), \bar{y}^{\prime \prime}(t, \alpha)\right), \\
& L\left[y^{\prime \prime}(t)\right]=\left\{p^{2} L[y(t)]-p y(t)\right\}-y^{\prime}(0)
\end{aligned}
$$

Therefore,

$$
L\left[f\left(t, y(t), y^{\prime}(t)\right)\right]=\left\{p^{2} L[y(t)]-p y_{0}\right\}-z_{0} .
$$

Hence,

$$
\begin{aligned}
& L\left[\underline{f}\left(t, y(t), y^{\prime}(t), \alpha\right)\right]=p^{2} L[\underline{y}(t, \alpha)]-p \underline{y}_{0}(\alpha)-\underline{z}_{0}(\alpha) \\
& L\left[\bar{f}\left(t, y(t), y^{\prime}(t), \alpha\right)\right]=p^{2} L[\bar{y}(t, \alpha)]-p \bar{y}_{0}(\alpha)-\bar{z}_{0}(\alpha) .
\end{aligned}
$$

where

$$
\begin{aligned}
& L\left[\underline{f}\left(t, y(t), y^{\prime}(t), \alpha\right)\right] \\
& =\min \left\{f(t, u, v), u \in(\underline{y}(t, \alpha), \bar{y}(t, \alpha)), v \in\left(\underline{y}^{\prime}(t, \alpha), \bar{y}^{\prime}(t, \alpha)\right)\right\}
\end{aligned}
$$

and

$$
\begin{aligned}
& L\left[\bar{f}\left(t, y(t), y^{\prime}(t), \alpha\right)\right] \\
& =\min \left\{f(t, u, v), u \in\left(\underline{y}(t, \alpha), \bar{y}^{\prime}(t, \alpha)\right), v \in\left(\underline{y}^{\prime}(t, \alpha), \bar{y}(t, \alpha)\right)\right\} .
\end{aligned}
$$

Therefore, the solution of equation (2) is obtained as

$$
\begin{aligned}
& L[\underline{y}(t, \alpha)]=H_{1}(p, \alpha), \\
& L[\bar{y}(t, \alpha)]=K_{1}(p, \alpha) .
\end{aligned}
$$


Taking the inverse Laplace transform of equation (7) and (8), the following are obtained:

$$
\underline{y}(t, \alpha)=L^{-1}\left[H_{1}(p, \alpha)\right]
$$

and

$$
\bar{y}(t, \alpha)=L^{-1}\left[K_{1}(p, \alpha)\right] .
$$

$H_{1}(p, \alpha)$ and $K_{1}(p, \alpha)$ are obtained using the assumption that the fuzzy linear function $f$ is given by $f\left(t, y(t), y^{\prime}(t)\right)=a y(t)+b y^{\prime}(t)+c(t)$, which is a crisp mapping and

$$
\begin{aligned}
& H_{1}(p, \alpha)=L[\underline{y}(t, \alpha)]=\frac{(p-b) \underline{y}_{0}(\alpha)+\underline{z}_{0}(\alpha)+L[c(t)]}{p^{2}-b p-a}, \\
& K_{1}(p, \alpha)=L[\bar{y}(t, \alpha)]=\frac{(p-b) \bar{y}_{0}(\alpha)+\bar{z}_{0}(\alpha)+L[c(t)]}{p^{2}-b p-a} .
\end{aligned}
$$

\section{Concept of gH-Differentiability}

Let $u, v \in F_{c}$, if there exists $\omega \in F_{c}$ such that $u=v+\omega$ then we say that there exists the $H_{\text {- }}$ difference between $u$ and $v$ which is denoted by $u-H, v=\omega$. Let $\left(\tau_{1}, \tau_{2}\right)$ be an open interval and let $t_{0} \in\left(\tau_{1}, \tau_{2}\right)$. Given $X:\left(\tau_{1}, \tau_{2}\right) \rightarrow F_{c}$ a fuzzy valued function, we say that $X$ is strongly generalized differentiable ( $G$ - differentiable) at $t_{0}$ if there exists an element $X^{\prime}\left(t_{0}\right) \in F_{c}$ such that

(i) for all $h>0$ sufficiently small,

$\exists X\left(t_{0}+h\right)-{ }_{H} X\left(t_{0}\right), X\left(t_{0}\right)-{ }_{H} X\left(t_{0}-h\right)$

and the limit in the metric $D$

$$
\lim _{h \rightarrow 0^{+}} \frac{X\left(t_{0}\right)-{ }_{H} X\left(t_{0}+h\right)}{-h}=\lim _{h \rightarrow 0^{+}} \frac{X\left(t_{0}-h\right)-{ }_{H} X\left(t_{0}\right)}{-h}=X^{\prime}\left(t_{0}\right)
$$

(ii) for all $h>0$ sufficiently small,

$\exists X\left(t_{0}+h\right)-{ }_{H} X\left(t_{0}\right), X\left(t_{0}\right)-{ }_{H} X\left(t_{0}-h\right)$

and the limit in the metric $D$ 


$$
\lim _{h \rightarrow 0^{+}} \frac{X\left(t_{0}+h\right)-{ }_{H} X\left(t_{0}\right)}{h}=\lim _{h \rightarrow 0^{+}} \frac{X\left(t_{0}\right)-{ }_{H} X\left(t_{0}+h\right)}{h}=X^{\prime}\left(t_{0}\right)
$$

(iii) for all $h>0$ sufficiently small,

$\exists X\left(t_{0}+h\right)-{ }_{H} X\left(t_{0}\right), X\left(t_{0}\right)-{ }_{H} X\left(t_{0}-h\right)$

and the limit in the metric $D$

$$
\lim _{h \rightarrow 0^{+}} \frac{X\left(t_{0}+h\right)-{ }_{H} X\left(t_{0}\right)}{h}=\lim _{h \rightarrow 0^{+}} \frac{X\left(t_{0}-h\right)-{ }_{H} X\left(t_{0}\right)}{-h}=X^{\prime}\left(t_{0}\right) \quad \text {; or }
$$

(iv) for all $h>0$ sufficiently small,

$$
\exists X\left(t_{0}+h\right)-{ }_{H} X\left(t_{0}\right), X\left(t_{0}\right)-{ }_{H} X\left(t_{0}-h\right)
$$

and the limit in the metric $D$

$$
\lim _{h \rightarrow 0^{+}} \frac{X\left(t_{0}\right)-{ }_{H} X\left(t_{0}+h\right)}{-h}=\lim _{h \rightarrow 0^{+}} \frac{X\left(t_{0}\right)-{ }_{H} X\left(t_{0}-h\right)}{h}=X^{\prime}\left(t_{0}\right)
$$

\section{Laplace Transform Method for Second Order FODE}

Suppose that the derivative $D$ takes a differentiable function $f$ defined on some interval $(a, b)$ and assigns to it a new function $D f=f^{\prime}$. The integral $I$ takes a continuous function $f$ defined on some interval $[a, b]$ and assigns to it a new function $I f(x)=\int_{a}^{x} f(t) d t$. The multiplication operator ${ }^{M_{\varphi}}$, which multiplies any given function $f$ on the interval $[a, b]$ by a fixed function $\varphi$ on $[a, b]$, is a transform

$$
\begin{aligned}
& M_{\varphi} f(x)=\varphi(x) \cdot f(x) \text {. The Laplace transform of the derivatives is } \\
& L\left[y^{\prime}\right]=\int_{0}^{\infty} e^{-p x} y^{\prime}(x) d x
\end{aligned}
$$

and in general,

$$
\begin{aligned}
L\left[f^{n}(t)\right]=s^{n} f(s)-s^{n-1} f(0)-s^{n-2} f^{\prime}(0)-s^{n-3} & f^{\prime \prime}(0) \\
& \quad-s^{n-4} f^{\prime \prime \prime}(0)-\cdots-f^{n-1}(0) .
\end{aligned}
$$


Lemma 1: If $\alpha=\min \left(a, \frac{b}{m}\right)$, then the successive approximations $y_{0}(t)=y_{0}$, therefore, $y_{k+1}(t)=y_{0}+\int_{t_{0}}^{t} f\left(y_{k}(s), s\right) d s$

are well defined in the interval $I=\left\{t|| t-t_{0} \mid \leq \alpha\right\}$, and on this interval

$\left|y_{k}(t)-y_{0}\right|<M\left|t-t_{0}\right| \leq b$

where $|f|<M$

Lemma 2: If $f$ and its derivatives belong to $C^{3}(R)$ then $y_{k}(t)$ in the successive approximations converges on $I$ to a solution of the differential equation (1) that satisfies the initial condition (2).

Lemma 3: Suppose that $f$ is continuous on a domain $R$ of the ${ }^{(t, x)}$ plane defined for $a, b>0$ , by $R=\left\{(t, x):\left|t-t_{0}\right| \leq a,\left\|x-x_{0}\right\| \leq b\right\}$, and that $f$ is Lipschitz in $x$ on $R$.

$M=\sup _{(t, x) \in R}\|f(t, x)\|<\infty \quad \alpha=\min \left(a, \frac{b}{M}\right)$.

Then the sequence defined by

$\phi_{0}=x_{0},\left|t-t_{0}\right| \leq \alpha$,

$\phi_{i}=x_{0}+\int_{t_{0}}^{t} f\left(s, \phi_{i-1}(s)\right) d s i \geq 1,\left|t-t_{0}\right| \leq \alpha$

converges uniformly on the interval $\left|t-t_{0}\right| \leq \alpha$ to $\phi$.

\section{RESULTS}

Establishing Results of Second Order FODE by Generalized Triangular Fuzzy Number (GTFN)

Solution of second order ODE (1) by FLT with $\gamma_{1}=\gamma_{3}, \omega=1_{\text {or }} \gamma_{2}=\gamma_{4}$ such that $\tilde{A}=\left(\gamma_{1}, \gamma_{2}, \gamma_{4}, \omega\right)$ or $\left(\gamma_{1}, \gamma_{3}, \gamma_{4}, \omega\right)$ 
and membership function

$$
\mu_{\tilde{A}}(x)=\left\{\begin{array}{l}
\omega \frac{x-\gamma_{1}}{\gamma_{2}-\gamma_{1}} \text { if } \gamma_{1} \leq x \leq \gamma_{2} \\
\omega \frac{\gamma_{1}-x}{\gamma_{4}-\gamma_{2}} \text { if } \gamma_{2} \leq x \leq \gamma_{4} \\
0, \text { otherwise }
\end{array}\right. \text {. }
$$

Now consider the FODE below

$$
\frac{d x_{i}(t, \alpha)}{d t}=k x_{i}(t, \alpha)+a \text { for } i=1,2 .
$$

where $a$ and $k$ are constant. The general solution of equation (14) is a fuzzy number with $\alpha$ -cut

$$
\begin{aligned}
& x_{1}(t, \alpha)=-\frac{\varepsilon}{k}+\left[\frac{\varepsilon}{k}+\left(\gamma_{1}+\frac{\alpha l_{\bar{\gamma}_{0}}}{\omega}\right)\right] e^{k\left(t-t_{0}\right)} \\
& x_{2}(t, \alpha)=-\frac{\varepsilon}{k}+\left[\frac{\varepsilon}{k}+\left(\gamma_{3}-\frac{\alpha r_{\bar{\gamma}_{0}}}{\omega}\right)\right] e^{k\left(t-t_{0}\right)}
\end{aligned}
$$

where $l_{\gamma_{0}}=\gamma_{2}-\gamma_{1}$ and $r_{\gamma_{0}}=\gamma_{3}-\gamma_{2}$.

Also consider the FODE

$$
\begin{aligned}
& \frac{d^{2} x_{1}(t, \alpha)}{d t^{2}}=-m x_{2}(t, \alpha) \\
& \frac{d^{2} x_{2}(t, \alpha)}{d t^{2}}=-m x_{1}(t, \alpha)
\end{aligned}
$$

Solving equations (17) and (18) respectively, the general solution is obtained as

$$
\begin{aligned}
& x_{1}(t, \alpha)=d_{1} e^{\sqrt{m} t}+d_{2} e^{-\sqrt{m} t}+d_{3} \sin \sqrt{m} t+d_{4} \cos \sqrt{m} t \\
& x_{2}(t, \alpha)=-d_{1} e^{\sqrt{m} t}-d_{2} e^{-\sqrt{m} t}+d_{3} \sin \sqrt{m} t+d_{4} \cos \sqrt{m} t .
\end{aligned}
$$

Applying the following conditions on equations (19) and (20), we have

$$
x_{1}(t, \alpha)=-\frac{\varepsilon}{k}+\frac{\varepsilon}{k} e^{k\left(t-t_{0}\right)}+\left(\gamma_{1}+\frac{\alpha l_{\gamma_{0}}}{\omega}\right) e^{k\left(t-t_{0}\right)},
$$




$$
\begin{aligned}
& x_{2}(t, \alpha)=-\frac{\varepsilon}{k}+\frac{\varepsilon}{k} e^{k\left(t-t_{0}\right)}+\left(\gamma_{3}-\frac{\alpha r_{\gamma_{0}}}{\omega}\right) e^{k\left(t-t_{0}\right)}, \\
& \frac{d x_{1}(t, \alpha)}{d t}=\frac{\varepsilon}{k} e^{-k t_{0}}+\left(\gamma_{1}+\frac{\alpha l_{\gamma_{0}}}{\omega}\right) e^{-k t_{0}}, \\
& \frac{d x_{2}(t, \alpha)}{d t}=\frac{\varepsilon}{k} e^{-k t_{0}}+\left(\gamma_{3}-\frac{\alpha r_{\gamma_{0}}}{\omega}\right) e^{-k t_{0}} .
\end{aligned}
$$

Substituting the conditions in equations (21), (22), (23) and (24) into equations (19) and (20) respectively, we have

$$
\begin{aligned}
& -\frac{\varepsilon}{k}+\frac{\varepsilon}{k} e^{k\left(t-t_{0}\right)}+\left(\gamma_{1}+\frac{\alpha l_{\gamma_{0}}}{\omega}\right) e^{k\left(t-t_{0}\right)}=d_{1} e^{\sqrt{m} t}+d_{2} e^{-\sqrt{m} t}+d_{3} \sin \sqrt{m} t+d_{4} \cos \sqrt{m} t, \\
& -\frac{\varepsilon}{k}+\frac{\varepsilon}{k} e^{k\left(t-t_{0}\right)}+\left(\gamma_{3}-\frac{\alpha r_{\gamma_{0}}}{\omega}\right) e^{k\left(t-t_{0}\right)}=-d_{1} e^{\sqrt{m} t}-d_{2} e^{-\sqrt{m} t}+d_{3} \sin \sqrt{m} t+d_{4} \cos \sqrt{m} t \\
& \frac{1}{\sqrt{m}}\left[\frac{\varepsilon}{k} e^{-k t_{0}}+\left(\gamma_{1}+\frac{\alpha l_{\gamma_{0}}}{\omega}\right) e^{-k t_{0}}\right]=d_{1} e^{\sqrt{m} t}+d_{2} e^{-\sqrt{m} t}+d_{3} \sin \sqrt{m} t+d_{4} \cos \sqrt{m} t \\
& \frac{1}{\sqrt{m}}\left[\frac{\varepsilon}{k} e^{-k t_{0}}+\left(\gamma_{3}-\frac{\alpha r_{\gamma_{0}}}{\omega}\right) e^{-k t_{0}}\right]=-d_{1} e^{\sqrt{m} t}-d_{2} e^{-\sqrt{m} t}+d_{3} \sin \sqrt{m} t+d_{4} \cos \sqrt{m} t
\end{aligned}
$$

Solving equations (25), (26), (27) and (28) respectively, we have

$$
\begin{aligned}
&\left(\gamma_{1}+\frac{\alpha l_{\gamma_{0}}}{\omega}\right) e^{k\left(t-t_{0}\right)}-\left(\gamma_{3}-\frac{\alpha r_{\gamma_{0}}}{\omega}\right) e^{k\left(t-t_{0}\right)}=2 d_{1} e^{\sqrt{m} t}+2 d_{2} e^{-\sqrt{m} t}, \\
& \frac{1}{\sqrt{m}}\left[\frac{\varepsilon}{k} e^{-k t_{0}}+\left(\gamma_{1}+\frac{\alpha l_{\gamma_{0}}}{\omega}\right) e^{-k t_{0}}\right]-\frac{1}{\sqrt{m}}\left[\frac{\varepsilon}{k} e^{-k t_{0}}+\left(\gamma_{3}-\frac{\alpha r_{\gamma_{0}}}{\omega}\right) e^{-k t_{0}}\right] \\
&=2 d_{1} e^{\sqrt{m} t}-2 d_{2} e^{-\sqrt{m} t} .
\end{aligned}
$$

Solving equations (29) and (30) respectively, we have

$$
d_{1}=\frac{1}{4}\left[\left(\gamma_{1}-\gamma_{3}+\left(\frac{\alpha\left(l_{\gamma_{0}}-r_{\gamma_{0}}\right)}{\omega}\right)\right) e^{k\left(t-t_{0}\right)}+\frac{1}{\sqrt{m}}\left(\gamma_{1}-\gamma_{3}+\left(\frac{\alpha\left(l_{\gamma_{0}}+r_{\gamma_{0}}\right)}{\omega}\right)\right) e^{-k t_{0}}\right] e^{-\sqrt{m} t_{0}},
$$




$$
d_{2}=\frac{1}{4}\left[\left(\gamma_{1}-\gamma_{3}+\left(\frac{\alpha\left(l_{\gamma_{0}}+r_{\gamma_{0}}\right)}{\omega}\right)\right) e^{k\left(t-t_{0}\right)}-\frac{1}{\sqrt{m}}\left(\gamma_{1}-\gamma_{3}+\left(\frac{\alpha\left(l_{\gamma_{0}}+r_{\gamma_{0}}\right)}{\omega}\right)\right) e^{-k t_{0}}\right] e^{\sqrt{m} t_{0}} \text {. }
$$

Also, from equation (25), (26), (27) and (28), we have

$$
\begin{aligned}
-\frac{2 \varepsilon}{k}+\frac{2 \varepsilon}{k} e^{k\left(t-t_{0}\right)}+\left(\gamma_{1}+\frac{\alpha l_{\gamma_{0}}}{\omega}\right) e^{k\left(t-t_{0}\right)}+ & \left(\gamma_{3}-\frac{\alpha r_{\gamma_{0}}}{\omega}\right) e^{k\left(t-t_{0}\right)} \\
& =2 d_{3} \sin \sqrt{m} t_{0}+2 d_{4} \cos \sqrt{m} t_{0}, \\
\frac{1}{\sqrt{m}}\left[\frac{\varepsilon}{k} e^{-k t_{0}}+\left(\gamma_{1}+\frac{\alpha l_{\gamma_{0}}}{\omega}\right) e^{-k t_{0}}\right]+\frac{1}{\sqrt{m}}[ & \left.\frac{\varepsilon}{k} e^{-k t_{0}}+\left(\gamma_{3}-\frac{\alpha r_{\gamma_{0}}}{\omega}\right) e^{-k t_{0}}\right] \\
& =2 d_{3} \sin \sqrt{m} t_{0}-2 d_{4} \cos \sqrt{m} t_{0} .
\end{aligned}
$$

Solving equations (33) and (34), we have

$$
\begin{gathered}
d_{3}=-\frac{1}{2}\left[\left[\frac{-2 \varepsilon}{k}\left(1-e^{k\left(t-t_{0}\right)}\right)+\left(\gamma_{1}+\gamma_{3}+\left(\frac{\alpha\left(l_{\gamma_{0}}-r_{\gamma_{0}}\right)}{\omega}\right)\right) e^{k\left(t-t_{0}\right)}\right] \sin \sqrt{m} t_{0}\right] \\
+\left[\frac{1}{\sqrt{m}}\left(\frac{2 \varepsilon}{k} e^{-k t_{0}}+\left(\gamma_{1}+\gamma_{3}+\left(\frac{\alpha\left(l_{\gamma_{0}}-r_{\gamma_{0}}\right)}{\omega}\right) e^{-k t_{0}}\right)\right)\right] \cos \sqrt{m} t_{0} \\
\begin{aligned}
d_{4}=\frac{1}{2}\left[\left[\frac{-2 \varepsilon}{k}\left(1-e^{k\left(t-t_{0}\right)}\right)+\left(\gamma_{1}+\gamma_{3}+\left(\frac{\alpha\left(l_{\gamma_{0}}-r_{\gamma_{0}}\right)}{\omega}\right)\right) e^{k\left(t-t_{0}\right)}\right] \cos \sqrt{m} t_{0}\right] \\
-\left[\frac{1}{\sqrt{m}}\left(\frac{2 \varepsilon}{k} e^{-k t_{0}}+\left(\gamma_{1}+\gamma_{3}+\left(\frac{\alpha\left(l_{\gamma_{0}}+r_{\gamma_{0}}\right)}{\omega}\right) e^{k t_{0}}\right)\right)\right] \sin \sqrt{m} t_{0}
\end{aligned}
\end{gathered}
$$

This shows that $d_{1}, d_{2}, d_{3}$ and $d_{4}$ are solutions of equation (1) when $\omega=1, \gamma_{2}=\gamma_{4}$ or $\gamma_{1}=\gamma_{3}$.

\section{CONSTRUCTED EXAMPLES}

\section{Existence of Second Order Differential Equations}

Consider FODE (1). Applying Lemma 1 to equation (1), we have

$$
y_{0}^{\prime}(t)=y^{0}+\int_{t_{0}}^{t} f\left(s, y_{0}(s), y_{0}^{\prime}(s)\right) d s
$$


$y_{1}^{\prime}(t)=y^{1}+\int_{t_{0}}^{t} f\left(s, y_{1}(s), y_{1}^{\prime}(s)\right) d s$

Let $y_{0}^{\prime}(t)=y_{0}(t), y_{1}^{\prime}(t)=y_{1}(t),\left|t-t_{0}\right| \leq \alpha$

$\left\|y_{1}-y_{0}\right\|=\left\|\int_{t_{0}}^{t} f\left(s, y_{0}(s), y_{0}^{\prime}(s)\right) d s\right\| \leq M\left|t-t_{0}\right| \leq \alpha M \leq b$

Thus,

$\left\|y_{1}(s)-y_{0}(s)\right\| \leq b$

Hence,

$\left\|y_{2}-y_{0}\right\|=\left\|\int_{t_{0}}^{t} f\left(s, y_{1}(s), y_{1}^{\prime}(s)\right) d s\right\| \leq \int_{t_{0}}^{t}\left\|f\left(s, y_{1}(s), y_{0}^{\prime}(s)\right)\right\| d s \leq \alpha M \leq b$

All subsequent terms in the sequence can similarly be defined and by induction, for

$\left|t-t_{0}\right| \leq\left\|y_{k}(t)-y_{0}\right\| \leq \alpha M \leq b, k=1, \cdots, n$

Now for $\left|t-t_{0}\right| \leq \alpha$,

$$
\begin{aligned}
& \left\|y_{k+1}(t)-y_{k}(t)\right\|=\left\|y^{0}+\int_{t_{0}}^{t} f\left(s, y_{k}(s), y_{k}^{\prime}(s)\right) d s-y^{0}-\int_{t_{0}}^{t} f\left(s, y_{k-1}(s), y_{k-1}^{\prime}(s)\right) d s\right\| \\
& =\left\|\int_{t_{0}}^{t}\left(f\left(s, y_{k}(s), y_{k}^{\prime}(s)\right)-f\left(s, y_{k-1}(s), y_{k-1}^{\prime}(s)\right)\right) d s\right\| \leq L \int_{t_{0}}^{t}\left\|\left(y_{k}(s), y_{k}^{\prime}(s)\right)-\left(y_{k-1}(s), y_{k-1}^{\prime}(s)\right)\right\| d s
\end{aligned}
$$

where the inequality above results to the fact that $f$ is Lipschitz. We then prove that for all $k$

$$
\left\|y_{k+1}-y_{k}\right\| \leq b \frac{\left(L\left|t-t_{0}\right|\right)^{k}}{k !},\left|t-t_{0}\right| \leq \alpha \text {. }
$$

Indeed, equation (39) holds for $k=1$ as previously established. Assume that equation (39) holds for $k=n$, then

$$
\begin{aligned}
& \left\|y_{k+1}(t)-y_{k}(t)\right\| \\
& =\left\|y^{0}+\int_{t_{0}}^{t} f\left(s, y_{k}(s), y_{k}^{\prime}(s)\right) d s-y^{0}-\int_{t_{0}}^{t} f\left(s, y_{k-1}(s), y_{k-1}^{\prime}(s)\right) d s\right\|
\end{aligned}
$$




$$
\begin{aligned}
& \left\|y_{n+2}(t)-y_{n+1}(t)\right\| \\
& =\left\|\int_{t_{0}}^{t}\left(f\left(s, y_{n+1}(s), y_{n+1}^{\prime}(s)\right)-f\left(s, y_{n}(s), y_{n}^{\prime}(s)\right)\right) d s\right\| \\
& \leq \int_{t_{0}}^{t} L\left\|y_{n+1}(s), y_{n+1}^{\prime}(s)-y_{n}(s) y_{n}^{\prime}(s)\right\| d s \leq L \int_{t_{0}}^{t}\left\|\left(y_{k}(s), y_{k}^{\prime}(s)\right)-\left(y_{k-1}(s), y_{k-1}^{\prime}(s)\right)\right\| d s \\
& \leq \int_{t_{0}}^{t} L b \frac{\left(L\left|t-t_{0}\right|\right)^{n}}{n !} d s \leq\left. b \frac{L^{n+1}}{n !} \frac{\left|t-t_{0}\right|^{n+1}}{n+1}\right|_{s=t_{0}} ^{s=t} \leq b \frac{\left(L\left|t-t_{0}\right|\right)^{n+1}}{(n+1) !}\left|t-t_{0}\right| \leq \alpha
\end{aligned}
$$

Therefore, equation (39) holds for $k=1, \cdots, n$. Thus, for $N>n$, we have

$$
\begin{aligned}
& \left\|y_{N}(t)-y_{n}(t)\right\| \leq \sum_{k=n}^{N-1}\left\|y_{k+1}(t), y_{k+1}^{\prime}(t)-y_{k}(t), y_{k}^{\prime}(t)\right\| \\
& \leq \sum_{k=n}^{N-1} b \frac{\left(L\left|t-t_{0}\right|\right)^{k}}{k !} \leq b \sum_{k=n}^{N-1} \frac{(L \alpha)^{k}}{k !}
\end{aligned}
$$

Applying lemma 2 and lemma 3 to equation (41), the equation tends to zero an $n \rightarrow \infty$. Therefore, $\left\{y_{k}(t)\right\}$ converges uniformly to a function $y(t)$ on the interval $\left|t-t_{0}\right| \leq \alpha$. As the convergence is uniform, the limit function is continuous; moreover, $y\left(t_{0}\right)=y_{0}$. Indeed

$$
y_{N}(t)=y_{0}(t)+\sum_{k=n}^{N-1}\left(y_{k}(t), y_{k}^{\prime}(t)-y_{k-1}(t), y_{k-1}^{\prime}(t)\right) . \text { So, } \quad y_{N}(t)=y_{0}(t)+\sum_{k=1}^{\infty}\left(y_{k}(t)-y_{k-1}(t)\right)
$$

The fact that $y(t)$ is a solution of fuzzy differential equations follows from the following results. If a sequence of functions $\left\{y_{k}(t)\right\}$ converges uniformly and that $y_{k}(t)$ are continuous on the interval $\left|t-t_{0}\right| \leq \alpha$, then

$$
\begin{aligned}
& \lim _{n \rightarrow \infty} \int_{t_{0}}^{t}\left(y_{n}(s), y_{n}^{\prime}(s)\right) d s=\int_{t_{0}}^{t} \lim _{n \rightarrow \infty}\left(y_{n}(s), y_{n}^{\prime}(s)\right) d s \\
& y(t)=\lim _{n \rightarrow \infty}\left(y_{n}(s), y_{n}^{\prime}(s)\right)=y^{0}+\lim _{n \rightarrow \infty} \int_{t_{0}}^{t} f\left(s, y_{n-1}(s), y_{n-1}^{\prime}(s)\right) d s \\
& =y^{0}+\int_{t_{0}}^{t} \lim _{n \rightarrow \infty} f\left(s, y_{n-1}(s), y_{n-1}^{\prime}(s)\right) d s=y^{0}+\int_{t_{0}}^{t} f\left(s, y(s), y^{\prime}(s)\right) d s .
\end{aligned}
$$


This implies that

$$
y(t)=y^{0}+\int_{t_{0}}^{t} f\left(s, y(s), y^{\prime}(s)\right) d s \quad \text { for }\left|t-t_{0}\right| \leq \alpha
$$

as the integrand $f(t, y)$ is a continuous function, $y(t)$ is differentiable with respect to $t$, and $y^{\prime}(t)=f(t, y(t))$, so $y(t)$ is a solution of the second order fuzzy differential equations (1). This shows that there exists a solution $y(t)$ to equation (1).

\section{Uniqueness of Second Order Differential Equations}

Consider the second order linear fuzzy ordinary differential equation

$$
\begin{aligned}
& y^{\prime \prime}(t)=f\left(t, y(t), y^{\prime}\right), y(0)=y_{0}=\left(\underline{y}_{0}, \bar{y}_{0}\right), y^{\prime}(0)=z_{0}=\left(\underline{z}_{0}, \bar{z}_{0}\right) \\
& y^{\prime}(t)=y^{0}+\int_{t_{0}}^{t} f\left(s, y(s), y^{\prime}(s)\right) d s \\
& x^{\prime}(t)=x^{0}+\int_{t_{0}}^{t} f\left(s, x(s), x^{\prime}(s)\right) d s
\end{aligned}
$$

Now, subtracting equation (43) out of (42)

Let $y^{\prime}(t)=y(t)$ and $x^{\prime}(t)=x(t)$

where $x(t), y(t) \in D$ and therefore,

$$
y(t)-x(t)=y^{0}-x^{0}+\int_{t_{0}}^{t}\left[f\left(s, y(s), y^{\prime}(s)\right)-f\left(s, x(s), x^{\prime}(s)\right)\right] d s .
$$

Taking the norm of both sides of equation (44) and applying the Lipschitz condition, it follows that

$$
0 \leq\|y(t)-x(t)\| \leq\left\|y^{0}-x^{0}\right\|+\left\|\int_{t_{0}}^{t} L\right\| y(s), y^{\prime}(s)-x(s), x^{\prime}(s)\|d s\| .
$$

We then applying the Gronwall inequality to equation (45) for $k=0$ and $r(t)=\|y(t)-x(t)\|$. For $t_{0} \leq t \leq t_{0}+\alpha$, we get $0 \leq\|y(t)-x(t)\| \leq 0$, that is, $\|y(t)-x(t)\|=0$, thus $y(t)=x(t)$ for $t_{0} \leq t \leq t_{0}+\alpha$. Similarly, for $t_{0}-\alpha \leq t \leq t_{0},\|y(t)-x(t)\|=0$. Therefore, $y(t)=x(t)$ for $\left|t-t_{0}\right| \leq \alpha$. This shows that the solution $y(t)=x(t)$ to equation (1) is unique. 


\section{DISCUSSION}

The solution of second order FODE (1) was obtained by Eljaoui, Melliani and Chadli (2015) without considering the relationship between the $k^{\text {th }}$ derivatives and FLT of second order. This study addresses the above gaps by obtaining the results in equations (31), (32), (35) and (36). After obtaining the relation between the FLT of second order and its $k^{\text {th }}$ derivative, it is observed that the existence and uniqueness of solution of second order FODE needs to be established. To obtain the results for the existence of solutions of second order linear homogeneous ODES, we applied lemma (1) to equation (1) which yielded the result in equation (41). Also, the uniqueness of solutions of the second order FODE (1) were established using lemma (2) and (3) on equation (1) which yielded the results in equation (45).

\section{CONCLUSION}

In this research, a second order FODE (1) was solved using Laplace transform method in a fuzzy environment. The results obtained in this study addressed the limitations to the assumptions that the fuzzy linear function $f\left(t, y(t), y^{\prime}(t)\right)=a y(t)+b y^{\prime}(t)+c(t)$ is a crisp mapping as well as the relationship between the the FLT of a fuzzy functions and its $k^{\text {th }}$ derivatives for $k \geq 1$ identified in El Jaoui, Melliani and Chadli (2015) and tested as well using existence and uniqueness lemmas.

\section{REFERENCES}

Casasnovas, J. \& Rossell, F. (2005), Averaging Fuzzy Biopolymers, Fuzzy Sets and Systems, 152, 139-158, http://dx.doi.org/10.1016/j.fss.2004.10.019

ElJaoui, E., Melliani, S. \&Chadli, L. S. (2015), Solving Second-Order Fuzzy Differential Equations by the Fuzzy Laplace Transform Method, Springer journal of advances in difference equations, DOI 10.1186/s13662-015-0414

Guo, M. Xue, M. X. \& Li, X. R. (2003), Impulsive Functional Differential Inclusions and Fuzzy Population Models, Fuzzy Sets and Systems, 138: 601-615,

http://dx.doi.org/10.1016/S0165-0114(02)00522-5

Naschie, M. S. E. (2005), From Experimental Quantum Optics to Quantum Gravity via a Fuzzy-khler Manifold, Chaos, Solitons and Fractals, 25:969-977, http://dx.doi.org/10.1016/j.chaos.2005.02.028

Oberguggenberger, M. \& Pittschmann, S. (1999), Differential Equations with Fuzzy Parameters, Math. Mod. Syst., 5: 181-202

Seikkala, S. (1987), On the Fuzzy Initial Value Problem, Journal of Fuzzy Sets and Systems, Vol. 24, 319-330 